\title{
La centralité urbaine à Québec. Un grand projet en continuel renouvellement
}

\author{
Guy Mercier et Francis Roy ${ }^{1}$
}

\section{INTRODUCTION}

L'urbanisme a le constant souci de revoir la composition et la configuration de la ville afin de l'accorder au mieux aux aspirations du moment ${ }^{2}$. L'intérêt concerne tout particulièrement le centreville, lieu emblématique et cour névralgique de la vie urbaine. Ainsi, à l'exercice de planification d'une agglomération, s'en ajoute toujours un autre, tout aussi fondamental, portant plus spécifiquement sur le noyau même de cet ensemble. Le but étant que la centralité urbaine, vecteur majeur de la dynamique sociale, puisse s'y réaliser dans des conditions optimales ${ }^{3}$.

L'enjeu n'est pas anodin, puisqu'il en tient à la volonté et à la capacité des êtres humains de se rencontrer et de se rassembler pour collaborer ou pour partager une expérience. Aussi est-il avantageux que des sites soient aménagés à cette fin.
Telle est la destination du centre-ville. Et comme les conditions de la centralité urbaine sont changeantes, il importe d'en adapter continument l'aménagement afin qu'elle garde son attractivité et son efficacité. C'est pourquoi un centre-ville se définit non seulement par ce qu'il est réellement, mais aussi par les jugements qu'on y porte et par les projets urbanistiques qu'il inspire ${ }^{4}$.

L'objectif de cet article est de démontrer comment la conception de la centralité urbaine constitue un projet en constante évolution, en prenant l'exemple de la Ville de Québec. Notre approche empruntera donc une démarche historique car il appert que la centralité imaginée pour cette ville par les autorités publiques a été rythmée par quatre projets distincts, au cours des six dernières décennies.

\section{RÉÉQUIPER UN CENTRE-VILLE DEVENU DÉSUET (LES ANNÉES 1950 ET 1960)}

\section{Dans les années suivant la Seconde Guerre mondiale, en maintes villes d'Amérique du Nord, le centre-ville, généralement jugé désuet, fut voué, par un urbanisme optimiste et ambitieux, à une rénovation complète qui, au-delà des améliorations escomptées, symbolisait la modernisation même de la société.}

Dans les années suivant la Seconde Guerre mondiale, en maintes villes d'Amérique du Nord, le centre-ville, généralement jugé désuet, fut voué, par un urbanisme optimiste et ambitieux, à une rénovation complète qui, au-delà des améliorations escomptées, symbolisait la modernisation même de la société ${ }^{5}$. La Vieille Capitale ne faisait pas exception à la règle ${ }^{6}$. On estimait en effet que son centre-ville n'était plus en mesure d'assurer pleinement sa mission ${ }^{7}$. Aussi, il paraissait nécessaire de le reconfigurer en profondeur, pour qu'il s'ajustât aux nouveautés de son époque. De profonds changements sociaux commandaient, évaluait-on, une autre ville et une autre manière d'y vivre ${ }^{8}$. Devenue le mode de transport privilégié, l'automobile, en suggérant une mobilité individuelle facile et rapide, justifiait la construction d'autoroutes urbaines, l'étalement de la ville, la séparation des usages et leur implantation dans des zones réservées au sein de l'espace urbain ${ }^{9}$. Le centre-ville, dans ce contexte, perdait ses repères.

L'industrie, à une autre époque, y avait fleuri - à Saint-Roch tout particulièrement ${ }^{10}$, mais elle s'apprêtait à l'abandonner, lorgnant les parcs industriels accrochés l'un après l'autre au flanc des autoroutes qui, de plus en plus, quadrillaient la périphérie. Le commerce, depuis toujours part essentielle du centre-ville de Québec, succombait à la tentation des centres commerciaux qui, eux aussi, se tournaient vers le réseau autoroutier en expansion. Ainsi, les commerçants de la rue SaintJean, de la rue Saint-Joseph et du boulevard 
Charest cédaient-ils le pas à ceux qui se regrouperaient, dans un même immeuble doté d'un spacieux stationnement, sur le plateau de Sainte-Foy ou ailleurs en périphérie. L'habitat, dense et populeux, souffrait de la comparaison avec l'irrésistible banlieue pavillonnaire qui insufflait un formidable élan à la périurbanisation qui gagnait ce même plateau de Sainte-Foy (à l'ouest), tout comme les anciennes paroisses agricoles de Charlesbourg (au nord) et de la Côtede-Beaupré (à l'est).

À ces forces qui entrainaient le déclin du centreville, l'urbanisme n'opposa, à vrai dire, aucune résistance $^{11}$. Il les encourageait plutôt, puisqu'il y trouvait motif à rénover au complet le centreville $^{12}$. Car si ce dernier déclinait, il n'en perdait pas son utilité, sauf qu'il n'était plus en état d'assumer correctement son rôle. Aussi urgeait-il de le débarrasser de ses équipements et de ses bâtiments anciens et d'en implanter des nouveaux, afin qu'il remplit au mieux sa vocation ${ }^{13}$. Il restait que cette dernière devait être adaptée, puisque certains usages devaient y trouver place, alors que d'autres devaient en être exclus.

L'industrie, au premier chef, devait en être délogée $^{14}$. L'espace dont elle y disposait était trop exigu et ses opérations, bien que légitimes, gênaient, jugeait-on désormais, la cohabitation. En contrepartie, s'était imposée la volonté d'afficher, autant à des fins patriotiques que touristiques, la valeur patrimoniale de la Vieille Capitale, d'où l'intention de restaurer le Vieux-Québec et de démolir le secteur de la Place-Royale pour le reconstruire à l'ancienne ${ }^{15}$. Quant à la fonction résidentielle et à l'activité commerciale, il fallait les limiter et les spécialiser, puisqu'il était acquis que la majorité habiterait en banlieue. Sous l'impulsion d'un secteur tertiaire et d'un Étatprovidence en pleine croissance et pourvoyeurs de nombreux emplois, l'heure était aux affaires et aux services. Aussi, le centre-ville fut-il appelé à devenir, par sa transformation radicale, le siège et l'image de cette modernité : la basse ville se réservant à Saint-Roch la centralité du secteur privé et la haute ville, au sein de la colline parlementaire, celle du secteur public ${ }^{16}$.

Il importait également de connecter efficacement ce centre-ville recomposé et revigoré aux banlieues d'où, en grand nombre, venaient et s'en retournaient, soir et matin, les employés. C'est pourquoi le centre-ville devait-il être directement branché au réseau autoroutier et pourvu d'aires de stationnement, en même temps qu'il devait être doté de grands édifices pouvant accueillir tout le personnel nécessaire à l'essor du secteur tertiaire privé et public (voir la figure 1). Deux équipements symbolisaient tout particulièrement cette modernisation : en haute ville, le Complexe G, haute tour de béton destinée à la fonction publique; en basse ville, le mail Saint-Roch, qui couvrit d'un toit une grande partie de la rue Saint-Joseph pour la transformer en galerie marchande, à l'image des centres d'achats qui fleurissaient en banlieue.

\section{Figure 1 - Années 1950 et 1960 : un centre-ville à moderniser ${ }^{17}$}

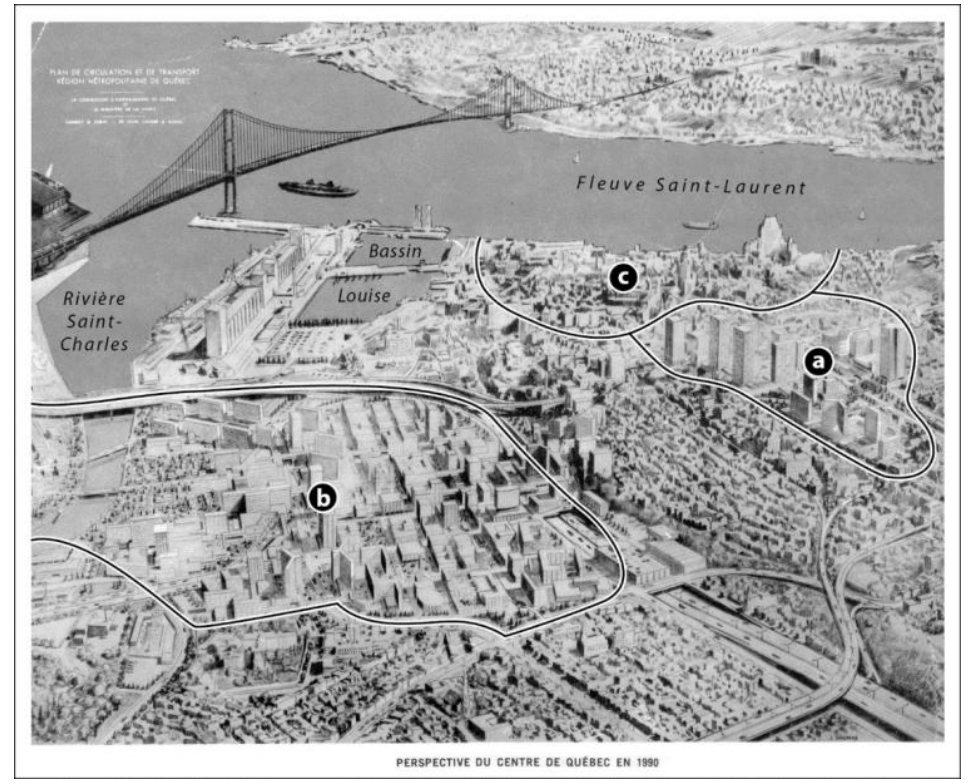




\section{STRUCTURER UNE CENTRALITÉ FRAGMENTÉE (LES ANNÉES 1970)}

\author{
Ainsi reconfiguré, le centre-ville était destiné \\ avant tout à ceux qui s'y rendaient pour \\ offrir ou pour recevoir des services, et moins \\ à ceux qui y habitaient. Sa raison d'être \\ résidait dans la place qu'il occupait dans un \\ espace urbain dont l'emprise devenait \\ régionale, phénomène que l'on nomme \\ aujourd'hui métropolisation.
}

Dans les années 1970, l'urbanisme québécois, animé du même espoir de modernisation, dut ajuster son projet de centralité urbaine à des contraintes et à des conséquences qui n'avaient pas été anticipées. En 1975, le premier schéma d'aménagement de la Communauté urbaine de Québec, créée en 1968, tenta d'ajuster le tir. Les vicissitudes de l'économie, les hésitations des autorités publiques et l'opposition des défenseurs de la population locale réduisaient les ambitions ou imposaient d'autres orientations. Parce qu'elle était plus ou moins achevée, cette rénovation marqua le centre-ville de Québec d'une profonde empreinte : les ménages s'y trouvaient moins nombreux et moins nantis; la centralité commerciale, quand elle subsistait, n'était plus son exclusivité; l'industrie l'avait déserté tandis que les cols blancs s'y concentraient encore davantage; l'automobile, pour laquelle il aurait fallu ajouter toujours plus de voies et de stationnements, y était envahissante ${ }^{18}$.

Ainsi reconfiguré, le centre-ville était destiné avant tout à ceux qui s'y rendaient pour offrir ou pour recevoir des services, et moins à ceux qui $\mathrm{y}$ habitaient. Sa raison d'être résidait dans la place qu'il occupait dans un espace urbain dont l'emprise devenait régionale, phénomène que l'on nomme aujourd'hui métropolisation ${ }^{19}$. L'aménagement des lieux publics favorisait en conséquence qu'on y arrive et qu'on en parte aisément, quitte à négliger le confort d'y être et le désir d'y rester. Or, une telle manière de concevoir le renouvellement du centre-ville, si elle pouvait motiver l'action, ne manquait pas, d'un autre point de vue, d'inspirer des entreprises concurrentes. En effet, le programme auquel on soumettait le centre-ville ancien pouvait aussi bien - et peut-être encore mieux - se réaliser ailleurs ${ }^{20}$.

Ce fut au reste ce qui arriva, puisqu'il se trouvât, dans les secteurs récemment urbanisés (SainteFoy, Charlesbourg et Beauport), des lieux - SainteFoy au premier chef - propices à générer la nouvelle centralité recherchée. Les carrefours autoroutiers et les centres commerciaux en favorisaient l'apparition. Quand la conjoncture s'y prêtait, un pôle d'emplois s'y consolidait et, du coup, un centre-ville surgissait sur un site où, il y a peu, s'étendait le domaine agricole ou la forêt. $\mathrm{Ne}$ pouvant nier l'évidence, l'urbanisme, comme il le faisait pour l'ancien centre-ville, le prit donc sous son aile et s'ingénia à définir la place de l'un et de l'autre dans la structure organisant la ville régionale en formation ${ }^{21}$. Si le but était de préciser le rôle de chacun et de rechercher la meilleure complémentarité entre eux, il restait que, dans les faits, le bénéfice de l'un était souvent ce que l'autre avait perdu ou ce qu'il eût pu obtenir.

\section{ASSUMER L'ÉCHEC DE LA RÉNOVATION DU CENTRE-VILLE (LES ANNÉES 1990)}

Le déploiement de la centralité au sein de pôles métropolitains, prévu au schéma d'aménagement de la Communauté urbaine de Québec de 1975, ne fut toutefois qu'un pis-aller sans commune mesure avec les difficultés rencontrées. Car si la modernisation urbanistique de Québec avait enclenché de grands changements, les bienfaits escomptés n'étaient pas tous au rendez-vous. Certes, le Vieux-Québec et le secteur de PlaceRoyale avaient réussi la modernisation qu'on avait espérée pour eux ${ }^{22}$. Leur statut patrimonial acquis en 1963 avait à la fois assuré leur transformation et leur conservation, de même qu'il avait affermi leur vocation touristique ${ }^{23}$. En revanche, les autres quartiers centraux - Saint-Roch notamment - ne présentaient pas un bilan reluisant. Les démolitions massives y avaient suscité une grogne et, parfois, une résistance farouche démontrant que tous n'appréciaient pas également l'œuvre engagée. Ainsi, l'urbanisme avait dû composer, depuis les années 1970, avec une population locale qui trouvait souvent à redire ${ }^{24}$. 
$\mathrm{Au}$ mécontentement et à l'opposition naissante, s'ajoutait un ralentissement économique. C'est pourquoi la reconstruction ne suivit pas toujours le rythme, laissant en plein cœur de ville de nombreux lots vacants et des bâtiments abandonnés ou presque en attente de démolition. Le centre-ville - le quartier Saint-Roch plus spécifiquement - prit du coup des airs d'inachèvement et de négligence qui connotaient à la fois une impuissance et une incompétence (voir la figure 2). Cette impression d'échec et d'abandon suscita une vive opposition à la politique de rénovation urbaine qui avait cours depuis les années 1950.
Elle se forma d'abord dans les rangs associatifs et fut ensuite prise en charge par un parti politique municipal qui, en 1989, remporta l'élection ${ }^{25}$. La priorité du nouveau gouvernement municipal fut de mettre en œuvre un urbanisme qui enfin pourrait offrir aux habitants de Saint-Roch une bonne qualité de vie. Pour ce faire, on estimait qu'il fallait que le quartier renouât avec son histoire $^{26}$. Alors que l'urbanisme rénovateur, au nom d'une prospérité à venir, avait porté atteinte au paysage traditionnel du quartier, le nouvel urbanisme comptait le préserver ${ }^{27}$.

Figure 2 - Un échec urbanistique : le « trou de Saint-Roch », c. $1980^{28}$

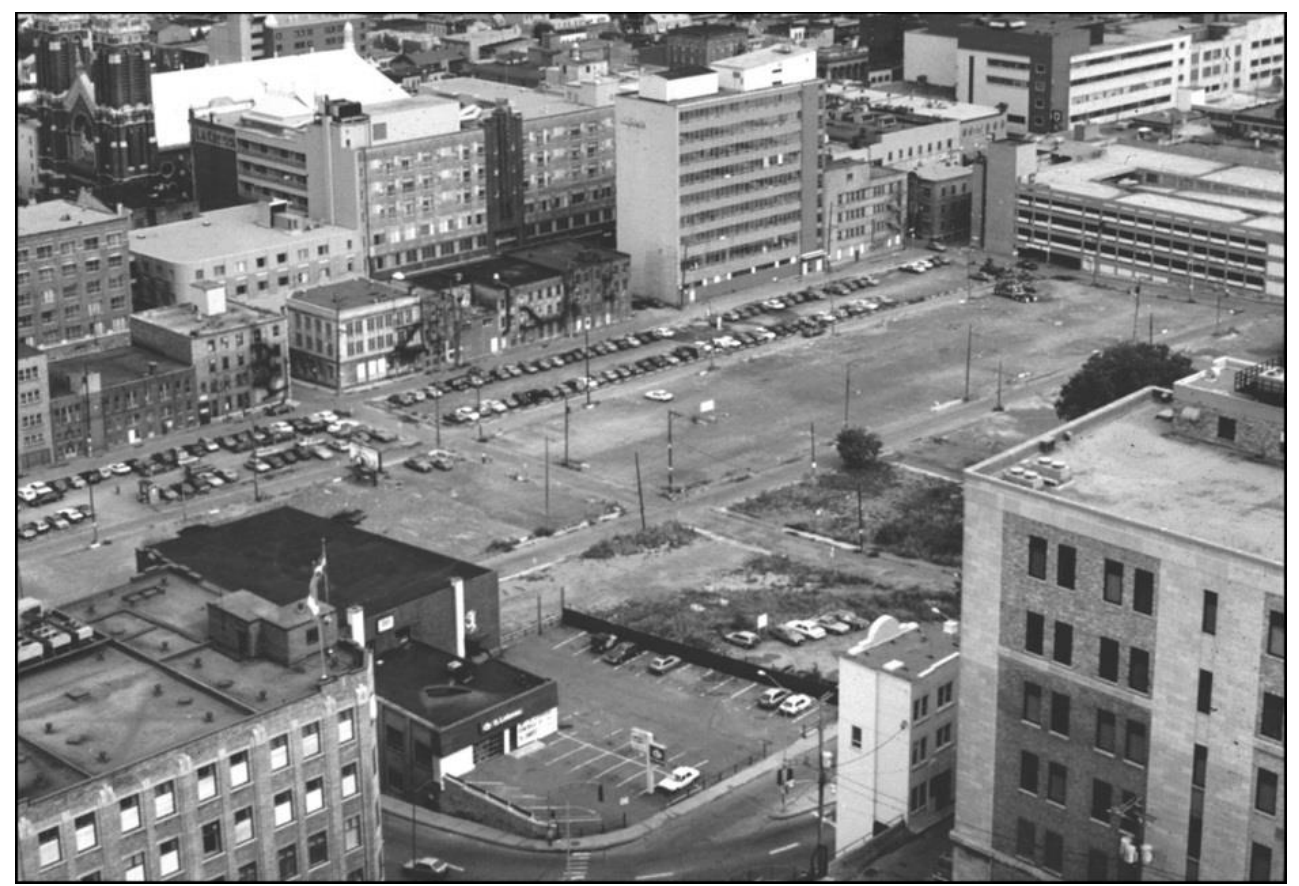

On voulait de la sorte montrer que l'urbanisme n'était pas une menace pour la population, mais une force positive contribuant à protéger et à améliorer leur milieu de vie (voir la figure 3 ). Il n'était pas interdit pour autant d'espérer que le quartier recouvrât une relative centralité économique, mais on s'assurait, en favorisant la conservation du bâti existant, que le renouveau du commerce et des affaires ne serait plus un motif de destruction. Au contraire, en insérant de nouvelles activités - de préférence associées à l'enseignement, à l'informatique et aux arts - dans des bâtiments anciens ou dans des bâtiments neufs respectueux de l'architecture ancienne, on voulait faire en sorte qu'une économie locale enfin revigorée confortât ce souci de préservation patrimoniale. Ce projet urbanistique devait de plus servir, au plan social, autant les résidents de longue date, souvent moins nantis et plus âgés, que les jeunes mieux pourvus et attirés par l'animation recouvrée $\mathrm{du}$ quartier ${ }^{29}$. Ainsi, Saint-Roch réactualisa sa centralité en accueillant derechef, dans un cadre bâti renouvelé ou restauré, investissements, emplois, commerces, chalandages, promeneurs, touristes et habitants. 
Figure 3 - Centralité et qualité de vie : le jardin Saint-Roch ${ }^{30}$

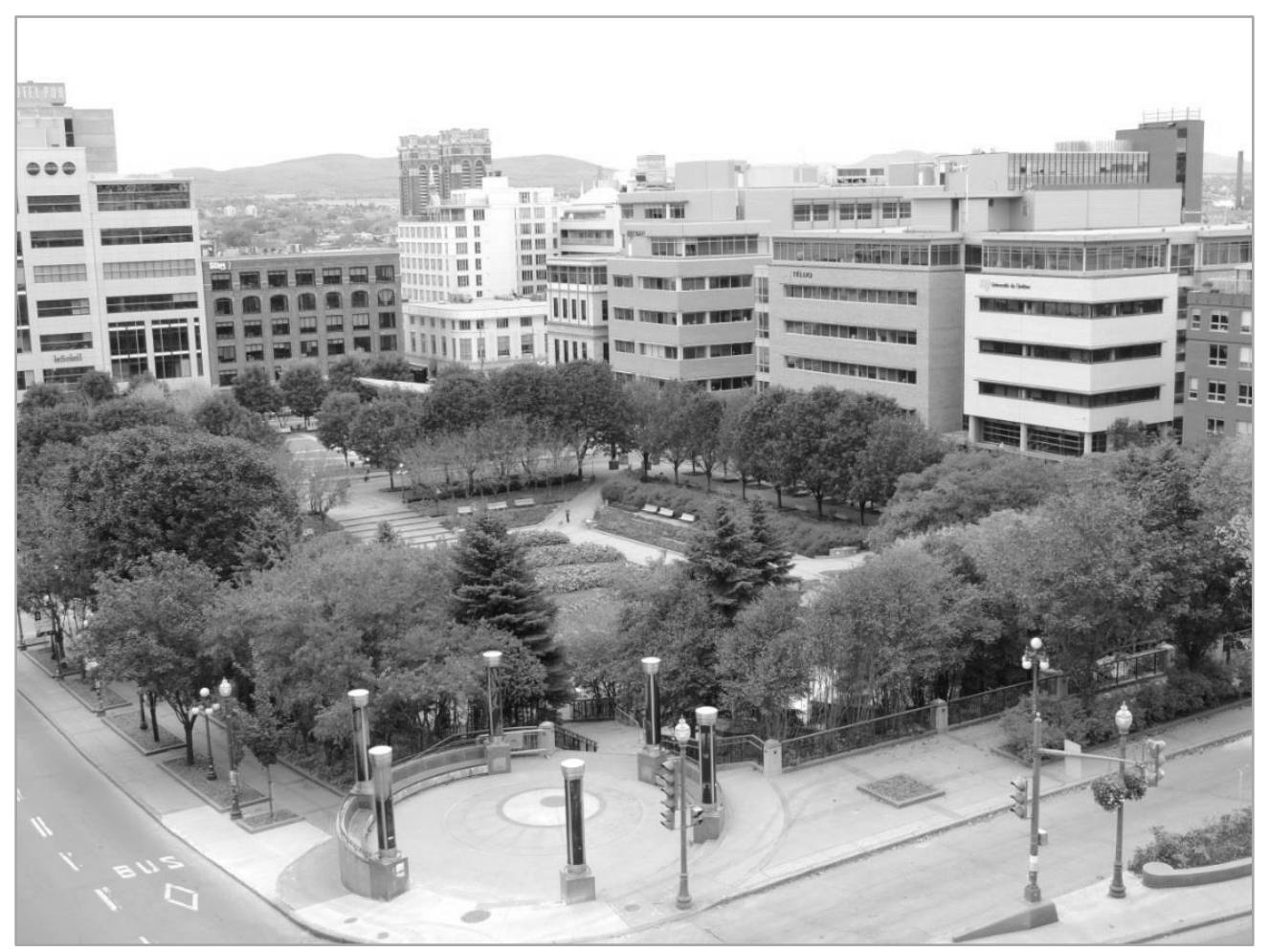

\section{LA CENTRALITÉ URBAINE FACE AU DÉFI DE LA DENSIFICATION (DES ANNÉES 2000 À AUJOURD'HUI)}

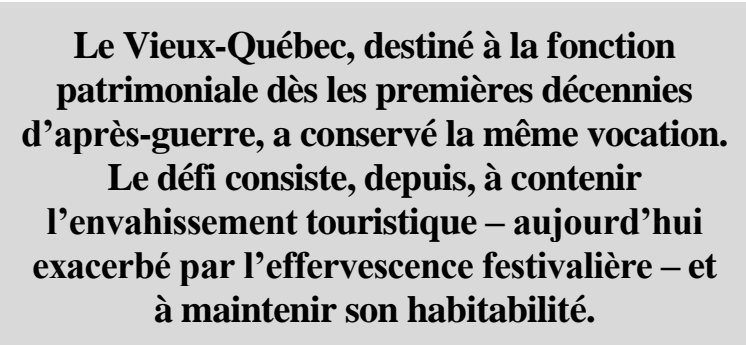

Si la centralité de Saint-Roch a été, à partir des années 1990, recomposée, elle demeure, à ce titre, une exception. En effet, pendant ce temps, les autres pôles qui, à Québec, assumaient aussi une part de centralité n'ont pas dérogé, ou si peu, au programme qui leur avait été respectivement assigné dans les périodes antérieures. Le VieuxQuébec, destiné à la fonction patrimoniale dès les premières décennies d'après-guerre, a conservé la même vocation. Le défi consiste, depuis, à contenir l'envahissement touristique - aujourd'hui exacerbé par l'effervescence festivalière - et à maintenir son habitabilité. La patrimonialité du secteur de PlaceRoyale n'a pas, elle non plus, été remise en cause, sinon qu'on a ajouté au cadre bâti, certes évocateur des temps anciens, mais sans réelle consistance sociale, des activités éducatives et récréatives qui confirment encore davantage le caractère muséal et touristique du lieu ${ }^{31}$.

La Colline Parlementaire, pour sa part, n'a pas dérogé à sa vocation institutionnelle. Seulement, il a été jugé nécessaire d'enrober les équipements modernes que les années 1970 lui avaient légués (complexes $\mathrm{G}$ et $\mathrm{H}$, voies encaissées du boulevard René-Lévesque, pénétration autoroutière jusqu'à l'hôtel du parlement), afin d'embellir les lieux et de les rendre plus attrayant ${ }^{32}$. La Commission de la Capitale nationale du Québec y a grandement contribué, en attendant que l'administration municipale y consacre, en 2010, un programme particulier d'urbanisme ${ }^{33}$. Le pôle de Sainte-Foy, de son côté, a accentué sa centralité d'affaires 
jusqu'à atteindre un point de saturation. Depuis son implantation dans les années 1960, commerces, services et autoroutes y ont côtoyé sans trop d'inconvénients un quartier résidentiel à dominante pavillonnaire. Mais la centralisation accentuant la pression, l'équilibre est aujourd'hui précaire. Ce centre-ville, sorti de terre dans la seconde moitié du $X^{\mathrm{e}}$ siècle, ne cessant de croitre, s'est ainsi imposé l'exigence de se projeter autrement sous l'angle urbanistique. Cette révision est devenue d'autant plus pertinente que, depuis 2000, SainteFoy, de même que plusieurs autres municipalités de banlieue, avait été fusionnée à la Ville de Québec. Aussi, est-il paru opportun de réexaminer la centralité urbaine à Québec selon cette nouvelle donne territoriale.

Le résultat de cette réflexion s'est d'abord manifesté dans le Plan directeur d'aménagement et de développement (PDAD), adopté par la Ville de Québec le 20 juin 2005. Y sont identifiés des secteurs nécessitant, en raison de leur haute centralité, une planification particulière, à la fois ambitieuse et détaillée. Depuis, il en a découlé l'élaboration et l'adoption de trois programmes particuliers d'urbanisme (PPU) relativement à la Colline Parlementaire (2010), au Plateau centre de
Sainte-Foy (2012) et à l'entrée de ville du quartier Saint-Roch $(2013)^{34}$. Dans les deux derniers cas tout spécialement, l'exercice a procédé d'une volonté de densification du territoire urbain, par une concentration des fonctions résidentielles, commerciales et institutionnelles.

En cherchant à inspirer et à coordonner divers projets, ces PPU constituent, à leur manière, autant de mégaprojets émanant d'une administration municipale en quête d'une nouvelle centralité urbaine. La grande envergure de l'entreprise se mesure d'une part aux efforts, aux énergies, aux ressources et au temps requis, autant de la part des autorités publiques, des agents économiques et des promoteurs immobiliers, que des groupes d'intérêt et des citoyens. D'autre part, elle se manifeste dans le gabarit même de plusieurs des équipements projetés, notamment un éventuel tramway appelé, dans les deux cas de Saint-Roch et de Sainte-Foy, à jouer un rôle structurant ${ }^{35}$. Les transformations envisagées doivent renforcer voire redéfinir la centralité urbaine au sein de l'agglomération de Québec, notamment par une affectation plus intense des fonctions résidentielles et commerciales (voir la figure 4).

\section{Figure 4 - Une centralité urbaine en quête de superficie d'occupation par la hauteur ${ }^{36}$}

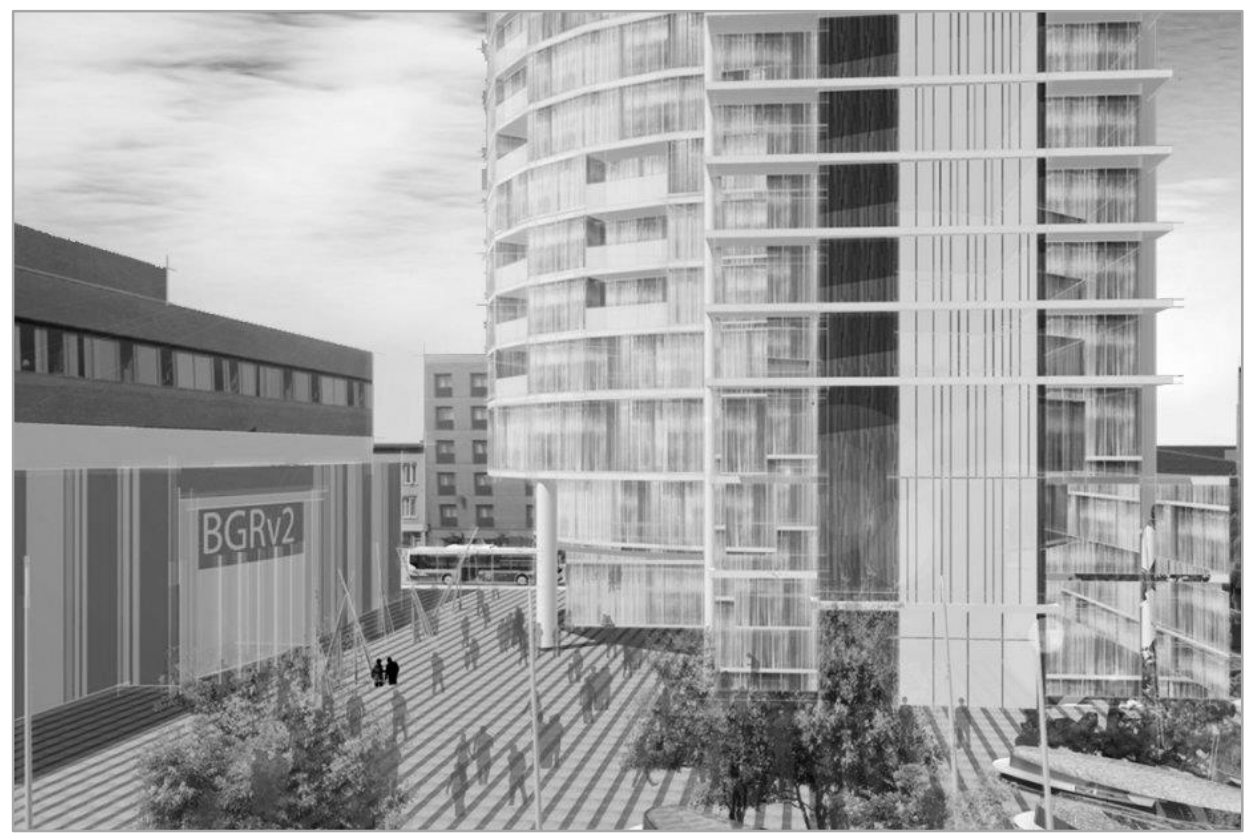


Au-delà des emplois, des services et des résidents que l'on souhaite y concentrer en plus grand nombre, ces secteurs doivent offrir une nouvelle image et commander une nouvelle perception. Or, ces mégaprojets font face à deux défis.

Le premier, à caractère sociologique, concerne l'adhésion de la population locale. La difficulté à ce titre tient autant à la qualité de vie des résidents qu'à la conception que ceux-ci se font de leur quartier. Par exemple, plusieurs citoyens du plateau centre de Sainte-Foy ont réalisé, lors des audiences publiques en vue de l'adoption du PPU de leur secteur, qu'ils ne vivaient plus désormais dans un quartier résidentiel, mais bien dans un centre-ville (principal pôle d'emplois du territoire de la Communauté métropolitaine de Québec). Bien que le plateau de Sainte-Foy constitue depuis longtemps déjà une place d'affaires très achalandée, la forme urbaine de cet ensemble - typique de la morphologie de la banlieue résidentielle d'après-guerre - peut suggérer le contraire. Or, ce projet, pourtant en continuité à bien des égards avec la situation antérieure, a soulevé des réactions vives de citoyens qui se voyaient du coup confrontés à une autre image de leur milieu de vie (voir la figure 5). Mais le problème n'est pas simplement affaire de perception. Car même s'ils peuvent, au fil du débat, adhérer aux grands objectifs de l'aménagement urbain, les citoyens ne s'en privent pas moins d'en contester l'application au moyen de normes de zonage sur leur bout de rue et les terrains avoisinants. Ainsi, la difficulté n'est pas simplement de faire partager une vision d'avenir de la ville, mais de trouver un équilibre entre la planification, qui exprime cette vision, et la qualité de vie, ponctuelle et actuelle, des citoyens du secteur concerné.

Le second défi porte sur la charge foncière qu'implique l'objectif de densification. Toutes proportions gardées, l'occupation de deux centres est passablement dense - davantage à Saint-Roch qu'à Sainte-Foy cependant - et la disponibilité foncière y reste limitée. Le sol et le mètre carré sont dans ce contexte des ressources rares. Pour y accéder, il faut définitivement rompre avec le modèle traditionnel d'occupation du sol à basse et moyenne densité dans ces secteurs. En plus d'une plus grande hauteur et profondeur de bâtiments (occupation des espaces aériens et souterrains), cela exige une colonisation des espaces vides $-\mathrm{du}$ moins que l'on peut considérer comme tels -, comme les stationnements de surface, usage typique en vigueur depuis quelques décennies, surtout dans les zones commerciales. Mais au-delà de la hauteur et de la surface d'occupation, il faut plus encore envisager une autre forme d'organisation de l'espace.

\section{Figure 5 - Une centralité qui s'affirme et s'affiche en contraste c $^{37}$}

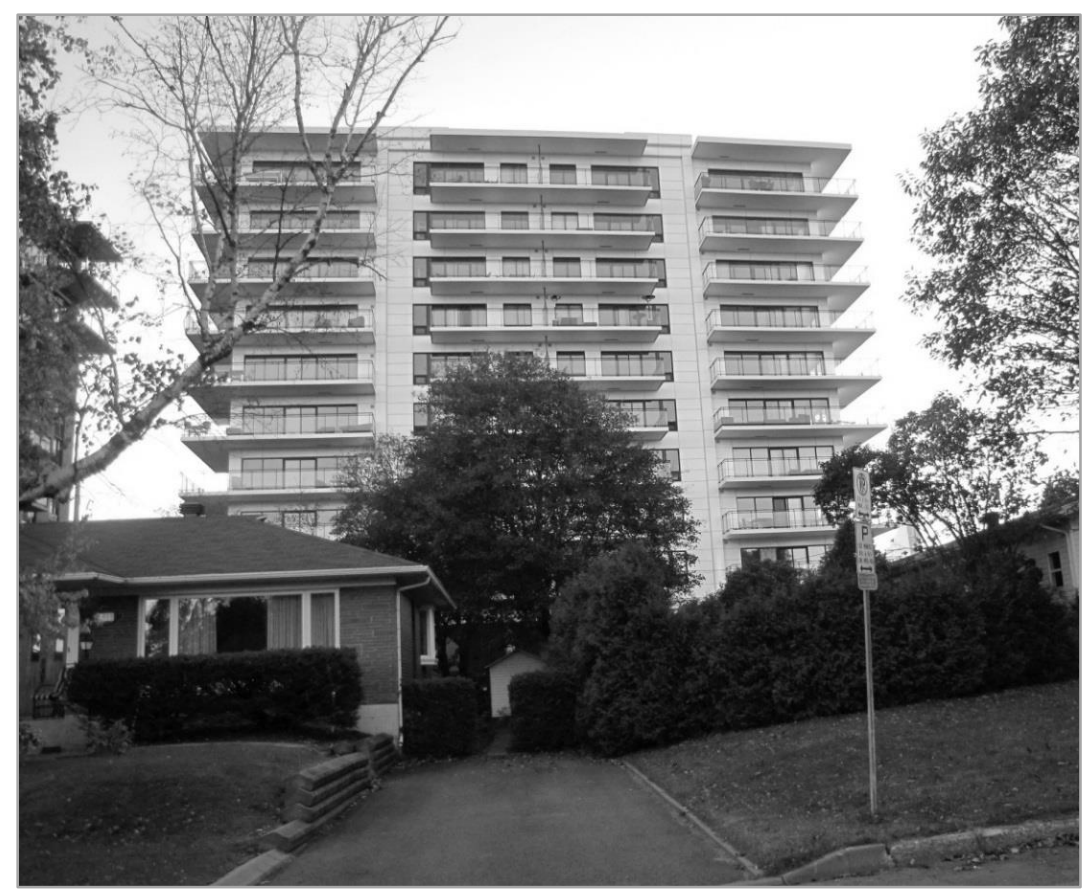


La relation entre le domaine bâti et le réseau viaire doit aussi être reconsidérée. L'implantation de bâtiments en bordure de rue devient dans ce contexte une nécessité, ce qui commande un autre traitement des façades et une autre conception de la circulation. Le chemin public, dans cette perspective, ne peut plus être réservé en priorité à la circulation motorisée, encore majoritairement automobile. D'axe de circulation mécanique (automobile, transport en commun, bicyclette), le chemin public doit, au risque de compromettre fluidité et débit, revêtir les atours d'un milieu de

\section{CONCLUSION}

La centralité urbaine de Québec est, depuis plus de cinquante ans, dans le collimateur de l'urbanisme. Suivant un contexte changeant, des projets se sont succédé pour l'accorder aux besoins et aux visions de chaque période.

Comme on le constate, la centralité urbaine de Québec est, depuis plus de cinquante ans, dans le collimateur de l'urbanisme. Suivant un contexte changeant, des projets se sont succédé pour l'accorder aux besoins et aux visions de chaque période. Mais dans son ensemble, la trajectoire marque un retournement concernant le rapport du centre urbain à sa périphérie. Au sortir de la Seconde Guerre mondiale, le centre-ville de Québec était sous le coup d'une évasion industrielle, commerciale et surtout résidentielle vers la banlieue. Le projet urbanistique, en soutien à ce mouvement, consista alors à lui réserver des fonctions spécifiques et à les répartir de manière à favoriser leur épanouissement. C'est pourquoi il apparut nécessaire de déployer la centralité en des pôles distincts, chacun assumant un rôle propre. $\mathrm{Si}$, au début, ces pôles (Vieux-Québec, Colline Parlementaire, Place-Royale et Saint-Roch) se jouxtaient au sein d'un unique centre-ville, il fallut bientôt, pour structurer un espace métropolitain en expansion, en ajouter d'autres au-delà du noyau initial, ce qui favorisa le remarquable essor du centre-ville de Sainte-Foy et, du coup, accéléra encore davantage la périurbanisation.

Aujourd'hui, en revanche, l'urbanisme ne conçoit plus la centralité comme un vecteur de l'étalement vie plus étoffé, propice à la circulation piétonne, voire à la flânerie et à la distraction, activités qui ne manquent pas d'intérêt commercial et que l'on peut concevoir comme essentielles à tout centreville digne de ce nom.

Bref, la densification ne présente pas qu'un aspect quantitatif et la création de la ressource foncière n'est pas seule en cause. S'y ajoute la nécessité d'une nouvelle approche urbanistique qui, en s'offrant elle aussi au débat, complexifie une problématique sociologique déjà bien délicate.

urbain, mais comme un facteur pouvant le contrecarrer, sinon le ralentir ou le limiter. Ce changement de perspective s'exprime d'une part à travers le projet urbanistique de densifier les pôles urbains centraux, du moins certains d'entre eux. En effet, l'intention de l'urbanisme, de 1950 à 1990, n'était pas d'accroitre l'usage du centre-ville de Québec. Il s'agissait plutôt d'y faire prospérer des usages qui lui étaient tout particulièrement appropriés. Le revirement concerne d'autre part la place réservée à la fonction résidentielle au centreville. Initialement, alors que l'habitat pavillonnaire s'imposait comme un idéal, il allait de soi de concevoir une centralité urbaine sans composante résidentielle déterminante. Désormais, l'urbanisme québécois favorise, dans l'optique d'une certaine mixité fonctionnelle et sociale, l'installation de nouveaux résidents dans les pôles centraux de Sainte-Foy et de Saint-Roch. Il reste à savoir si une telle réorientation suffira pour freiner l'étalement urbain.

Considérant l'élan que conserve toujours l'habitat pavillonnaire, on serait porté à en douter. Et si la mesure avait quelque effet, ne faut-il pas craindre, surtout si l'automobile maintient sa domination sur les transports, que se reproduise au centre-ville cette confusion des usages - certes selon une autre configuration, mais elle aussi avec un néfaste cortège de nuisances - que l'urbanisme d'aprèsguerre voulait tant dissiper? Bien sûr, il n'y a pas lieu, en urbanisme, d'être pessimiste, sinon à quoi bon d'y consentir temps, réflexion et effort. Une juste appréciation des expériences passées n'en appelle pas moins à la prudence. 


\section{BIBLIOGRAPHIE ET NOTES}

${ }^{1}$ Les auteurs sont professeurs à la Faculté de foresterie, géographie et géomatique de l'Université Laval.

${ }^{2}$ Hubert, M., Lewis, P. et Raynaud, M. M. (dir.) (2104). Les grands projets urbains. Territoires, acteurs et stratégies. Montréal, Presses de l'Université de Montréal.

${ }^{3}$ Isenberg, A. (2004). Downtown America. Chicago, University of Chicago Press.

${ }^{4}$ Strom, E. (2008). «Rethinking the politics of Downtown development », Journal of Urban Affairs, vol. $30, n^{\circ} 1$, pp. 37-61.

${ }^{5}$ Fogelson, R. M. (2001). Downtown: its rise and fall, 1880-1950, New Haven, Yale University Press.

${ }^{6}$ Vallières, M. et al. (2008). Histoire de Québec et de sa région, tome III : 1940-2008. Québec, Presses de l'Université Laval, p. 1610-2523; Blais, C., Gallichan, G., Lemieux, F. et Saint-Pierre, J. (2008). Québec, quatre siècles d'une capitale, Québec, Les Publications du Québec; Lebel, J.-M. et Roy, A. (2000). Québec 19002000. Le siècle d'une capitale, Québec, Éditions Multimondes et Commission de la capitale nationale du Québec, 159 p.; Hatvany, M. (2001). «L'expansion urbaine du $\mathrm{XX}^{\mathrm{e}}$ siècle », dans Serge Courville et Robert Garon (dir.) Québec, ville et capitale, Québec, Presses de l'Université du Québec, p. 256-279 et St-Hilaire, M. (2001). «Entre hier et demain », Dans Serge Courville et Robert Garon (dir.) Québec, ville et capitale, Québec, Presses de l'Université Laval, p. 330-333.

${ }^{7}$ Gréber, J., Fiset, É. et Bédard, R. (1956). Projet d'aménagement de Québec et de sa région, Québec, Ville de Québec et Leclerc, F. (2014) Savoirs et pouvoirs dans la ville de Québec : le Rapport Gréber et les images discursives de la modernité urbaine, 19481956. Québec, Université Laval, Département de géographie, mémoire de maitrise.

${ }^{8}$ Trotier, L. (1962-1963). Transformations récentes de l'agglomération québécoise : fonctions, population et organisation de l'espace. Cahiers de géographie de Québec, vol. 7, no 13, p. 7-27; Noppen, L. et Morisset, L. (1998a). Québec de roc et de pierres. La capitale en architecture, Québec, Éditions MultiMondes et Commission de la capitale nationale du Québec et Mercier, G. (2010). «La modernisation de Québec après la Seconde Guerre mondiale: une ville sous l'emprise de sa propre image », Dans Lucie K. Morisset et Marie-Ève Breton (dir.) La ville, phénomène de représentation, Québec, Presses de l'Université du Québec, p. 125-149.

${ }^{9}$ Plan de circulation et de transport. Région métropolitaine de Québec, Société d'ingénieursconseils Vandry \& Jobin - De Leuw, Caher et associés, 3 vol., 1967 et 1968 .

${ }^{10}$ Morisset, L. K. (2001). La mémoire du paysage. Histoire de la forme urbaine d'un centre-ville: SaintRoch, Québec, Québec, Presses de l'Université Laval.
${ }^{11}$ Fortin, A., Després, C. et Vachon, G. (2011). La banlieue s'étale, Québec, Nota Bene.

${ }^{12}$ Mercier, G. (2010). «La modernisation de Québec après la Seconde Guerre mondiale: une ville sous l'emprise de sa propre image », Dans Lucie K. Morisset et Marie-Ève Breton (dir.) La ville, phénomène de représentation, Québec, Presses de l'Université du Québec, p. 125-149.

${ }^{13}$ Filion, P. (1987). «Core Development, Neighbourhood Revitalization and Municipal Government Motivation: Twenty Years of Urban Renewal in Quebec City», Revue canadienne des sciences politiques, vol. 20, n ${ }^{\circ} 1$, p. 131-147.

${ }^{14}$ Martin, J.-M. et al. (1961-1963). Le logement à Québec; volume 1: Recommandations; volume 2: Caractéristiques générales de l'habitation à Québec; volume 3 : Les conditions d'habitation et la qualité des logements; volume 4: Prévisions. Québec, Ville de Québec.

${ }^{15}$ Mercier, G. (2003a). « La compétence de démolir. La ville de Québec à l'âge du réaménagement urbain », dans Frédéric Lasserre et Aline Lechaume (dir.) Le territoire pensé. Géographie des représentations territoriales, Québec, Presses de l'Université du Québec, p. 267-295 et FAURE, I. (1992). «La reconstruction de Place-Royale à Québec », Cahiers de géographie du Québec, vol. 36, nº 98, p. 321-336.

${ }^{16}$ Mercier, G. (2003b). « The Rhetoric of Contemporary Urbanism: A Deconstructive Analysis of Central City Neighborhood ", Canadian Journal of Urban Research, vol. 12, $\mathrm{n}^{\mathrm{o}}$ 1, p. 71-98.

${ }^{17}$ Aux yeux de l'urbanisme modernisateur, le centreville de Québec devait être constitué de trois pôles : la Colline Parlementaire (a), le quartier Saint-Roch (b) et le Vieux Québec/Place-Royale (c). Chacun de ces pôles devait porter une image spécifique de la ville: la gestion étatique efficace, l'économie prospère et l'histoire inspirante. Le tout constituant le point focal d'une nouvelle ville-région efficacement ventilée et bien connectée au reste du monde grâce à un réseau d'autoroutes approprié (Source: Plan de circulation et de transport. Région métropolitaine de Québec, 1968 : 24)

${ }^{18}$ Hulbert, F. (1994). Essai de géopolitique urbaine et régionale. La comédie urbaine de Québec, Montréal, Méridien.

${ }^{19}$ Bélanger, C., Mercier, G. et Bédard, M. (1999). «La structure urbaine de la région de Québec », dans Guy Mercier, Claude Bélanger et Mario Bédard (dir.) L'avenir municipal. Dynamiques québécoises et canadiennes. Québec, Éditions Sylvain Harvey, p. 1-35. ${ }^{20}$ Vallières, M. et al. (2008). Histoire de Québec et de sa région, tome III : 1940-2008. Québec, Presses de l'Université Laval, p. 1610-2523 et Hulbert, F. (1994). Essai de géopolitique urbaine et régionale. La comédie urbaine de Québec, Montréal, Méridien. 
${ }^{21}$ Communauté urbaine de Québec (1975). Schéma d'aménagement de la Communauté urbaine de Québec, Québec, Presses de 1'Université Laval.

${ }^{22}$ Voir COMMISSION DES BIENS CULTURELS DU QUÉBEC (2007). Empreintes et mémoires. L'arrondissement historique du Vieux-Québec, Québec, Les Publications du Québec; NOPPEN, L. (1993). «Place-Royale, chantier de construction d'une identité nationale », dans Patrick Dieudonné (dir.) Villes reconstruites. Du dessin au destin, Paris, L'Harmattan, vol. 2, pp. 301-306 et PROVENCHER, J. (2007). L'histoire du Vieux-Québec à travers son patrimoine, Québec, Les Publications du Québec.

${ }^{23}$ Voir BÉLANGER, M. et CIMON, J. (1990). «Le Centre-ville de Québec : espace sacré, ambiances, tourisme », dans Caroline Andrew et Louise Quesnel (dir.) L'aménagement des centres-villes, Ottawa, M Éditeurs, p. 143-154.

${ }^{24}$ Voir SIMARD, M. (2000). Développement local et identifié communautaire: l'exemple du quartier Saint-Roch à Québec, Cahiers de géographie $d u$ Québec, vol. 44, n 122, p. 167-188.

${ }^{25}$ Voir MERCIER, G. (2003b). «The Rhetoric of Contemporary Urbanism: A Deconstructive Analysis of Central City Neighborhood », Canadian Journal of Urban Research, vol. 12, n ${ }^{\circ}$ 1, p. 71-98.

${ }^{26}$ Voir NOPPEN, L. et MORISSET, L. K., dir. (1999). Québec, la renaissance d'un tout autre centre-ville, numéro thématique de ARQ. La revue d'architecture, $\mathrm{n}^{\mathrm{O}} 107$.

${ }^{27}$ Voir VILLE DE QUÉBEC (1990). Un Plan d'action pour le quartier Saint-Roch : document de consultation. Un quartier renouvelé pour une capitale renforcée.

${ }^{28} \mathrm{La}$ rénovation du quartier Saint-Roch, entreprise dans les années 1960, fut stoppée ou presque, laissant pendant longtemps ce vaste terrain en attente d'affectation, au point de renverser l'image : ce qui devait témoigner d'un indéniable progrès en fut réduit à attester de l'impuissance et de l'impasse de l'urbanisme modernisateur (Source: Collection particulière).

${ }^{29}$ Voir FREEDMAN, M. (2009). «Se reconnaître dans un centre-ville en revitalisation. Expériences d'itinérants dans le quartier Saint-Roch à Québec », ACME: An International E-Journal for Critical Geographies, vol. 8, $\mathrm{n}^{\circ}$ 1, p. 100-122.

${ }^{30}$ Sur le site de l'ancien «trou de Saint-Roch » apparut en 1992, au côté d'institutions publiques, de commerces et de logements, un jardin donnant une nouvelle tonalité au quartier (Photo : Guy Mercier, 2013).

${ }^{31}$ Voir BERTHOLD, É. (2012). Patrimoine, culture et récit. L'île d'Orléans et la place Royale de Québec. Québec, Presses de l'Université Laval.

${ }^{32}$ Voir NOPPEN, L. et MORISSET, L. K. (1998b). «L'urbanisme végétal: de l'usage historique et postmo-derne », dans Guy Mercier et Jacques
Bethemont (dir.) La ville en quête de nature, Québec et Lyon, Septentrion et Centre Jacques-Cartier, p. 211237.

${ }^{33}$ Le programme particulier d'urbanisme est, au Québec, un document de planification institué en vertu de la Loi sur l'aménagement et l'urbanisme. Ce document complète le plan d'urbanisme en s'attachant pour sa part à un secteur particulier du territoire municipal, tout en étant plus précis et plus détaillé.

${ }^{34}$ Ville de Québec (2010) Programme particulier d'urbanisme - Colline Parlementaire. [en ligne] http://www.ville.quebec.qc.ca/grandsprojetsverts/terr itoire/ppu_colline/index.aspx; Ville de Québec (2010) Programme particulier d'urbanisme - Entrée de ville du quartier Saint-Roch. [en ligne] http://www.ville.quebec.qc.ca/grandsprojetsverts/terr itoire/ppu_stroch/index.aspx et Ville de Québec (2013) Programme particulier d'urbanisme - Plateau de Sainte-Foy. [en ligne] http://www.ville.quebec.qc. ca/grandsprojetsverts/territoire/ppu_saintefoy/.

${ }^{35}$ Ville de Québec (2009). Vivre et se déplacer à Québec, Document de consultation en marge de l'élaboration d'un plan de mobilité durable.

${ }^{36}$ Le Programme particulier d'urbanisme du quartier Saint-Roch prévoit l'implantation d'une tour à vocation résidentielle et commerciale sur la place Jacques-Cartier. Sa hauteur excède celle de tous les immeubles du secteur. Le projet prévoit également une hausse des élévations dans les environs. Tel serait le prix à payer aujourd'hui pour conforter la centralité d'un milieu déjà densément construit (Source : TVA Nouvelles http://tvanouvelles.ca/lcn/ infos/regional/quebec/archives/2013/02/20130214125749.html.

${ }^{37}$ Le Programme particulier d'urbanisme du plateau de Sainte-Foy vise la densification d'un milieu où dominait depuis plusieurs décennies l'habitat pavillonnaire. Ce projet encourage la construction en hauteur qui, depuis quelques années maintenant, transforme profondément le paysage (Source: Francis Roy, 2013). 